\title{
Influences of Microporous Layer Design Parameters for Gas Diffusion Layer on Permeability and PEFC Performance*
}

\author{
Tatsumi KITAHARA**, Toshiaki KONOMI**, \\ Hironori NAKAJIMA** and Masato KAZAMA*** \\ ** Department of Mechanical Engineering, Kyushu University \\ 744 Motooka, Nishi-ku, Fukuoka, 819-0395, Japan \\ E-mail:kitahara@mech.kyushu-u.ac.jp \\ *** Graduate School of Engineering, Department of Mechanical Engineering, \\ Kyushu University, 744 Motooka, Nishi-ku, Fukuoka, 819-0395, Japan
}

\begin{abstract}
Gas diffusion layers (GDLs) coated with a microporous layer (MPL) have been commonly used to improve water management properties of polymer electrolyte fuel cells (PEFCs). However, the appropriate pore diameter, thickness and hydrophobicity of the MPL remain unclear. In the present study, the influences of MPL design parameters on permeability and PEFC performance were evaluated. A decrease in the pore diameter of the MPL reduces through-plane permeability significantly, but reduces in-plane permeability only slightly. Under high-humidity conditions, a decrease in the MPL pore diameter is effective for preventing flooding, enhancing PEFC performance. However, when the pore diameter becomes too small, PEFC performance tends to decrease. Reducing the MPL thickness improves in-plane permeability, enhancing the ability of the MPL to avoid flooding. Under low-humidity conditions, a decrease in the MPL pore diameter is effective for preventing drying-out of the MEA. Increasing the MPL thickness is also effective for maintaining the humidity of the MEA. However, when the MPL thickness becomes too large, the transport of oxygen to the electrode through the GDL is deteriorated, lowering PEFC performance.
\end{abstract}

Key words: Polymer Electrolyte Fuel Cell, Gas Diffusion Layer, Microporous Layer, Air Permeability, Pore Diameter, Flooding, Drying-out, Overpotential

\section{Introduction}

Polymer electrolyte fuel cells (PEFCs) with high efficiency and low environmental impact have been developed in recent years. However, further improvement in performance and reliability are required to ensure that PEFCs constitute a large proportion of future power sources. In order to enhance PEFC performance, appropriate water balance between the conservation of membrane humidity and the discharge of water produced in the cell is essential. Loss of water content in the membrane decreases ionic conductivity, thereby increasing the internal resistance of the cell. The basic configuration of a PEFC involves a membrane electrode assembly (MEA), a gas diffusion layer (GDL) and a separator. The design parameters for the GDL, such as thickness, pore size distribution, electrical resistivity, gas permeability and hydrophobicity, play an important role in determining the characteristics of electron transport, gas diffusion and water management during PEFC operation ${ }^{(1)}$.

*Received 22 Oct., 2010 (No. T1-09-0039) Japanese Original : Trans. Jpn. Soc. Mech. Eng., Vol.75, No.755, B (2009), pp.1524-1531 (Received 14 Jan., 2009) [DOI: 10.1299/jee.6.17]

Copyright $\odot 2011$ by JSME 
Several investigations have demonstrated that the GDL coated with a hydrophobic microporous layer (MPL) is effective for improvement of the water management characteristics, thereby enhancing PEFC performance ${ }^{(2)-(8)}$. The authors have also reported that the MPL coated GDL significantly reduces flooding on the electrode, enhancing PEFC performance under high humidity conditions ${ }^{(9)}$. The MPL is also effective to prevent drying-out of the MEA under low humidity conditions. The pore size, porosity, thickness and hydrophobicity of the MPL have a significant influence on the gas diffusion and water management characteristics of the GDL. Therefore, it is important to clarify appropriate design parameters of the MPL to enhance PEFC performance.

The present study was carried out to determine appropriate design parameters of the MPL coated GDL. The in-plane and through-plane gas permeability of the GDL was measured under compression conditions typical of PEFCs under operation. The maximum pore and mean flow pore diameters were evaluated from the permeability tests. The MPL thickness considering the penetration into the substrate was estimated by a comparison of the in-plane permeability obtained using GDLs with and without the MPL. The influences of the MPL design parameters on PEFC performance were also clarified under high and low humidity conditions.

\section{Experimental}

\subsection{Gas Permeability Test}

Figure 1 shows a schematic diagram of the GDL permeability test apparatus ${ }^{(9)}$. The GDL was placed between two cylindrical plates. A soft O-ring was used for gas sealing between the plates. The force required to deform the O-ring was negligible compared with the compression force acting on the GDL. The compression force was controlled using a clamp screw and was measured with a load cell. For the gas permeability tests, the compression pressure was set at 1 $\mathrm{MPa}$, as measured in a typical PEFC $^{(10)}$. Figure 2 shows the major dimensions of the GDL used for the through-plane and in-plane permeability tests. The supplied air pressure (gauge pressure) was set at $1.23 \mathrm{kPa}$, which is the same as that in the Gurley method ${ }^{(11)}$. The air flow rate $Q$ was measured using a mass flow meter. The permeance $q$ was defined as the flow rate divided by the supplied air pressure and permeable cross-sectional area (11). Maximum pore and mean flow pore diameters of the GDLs were measured as shown in Figure 3.

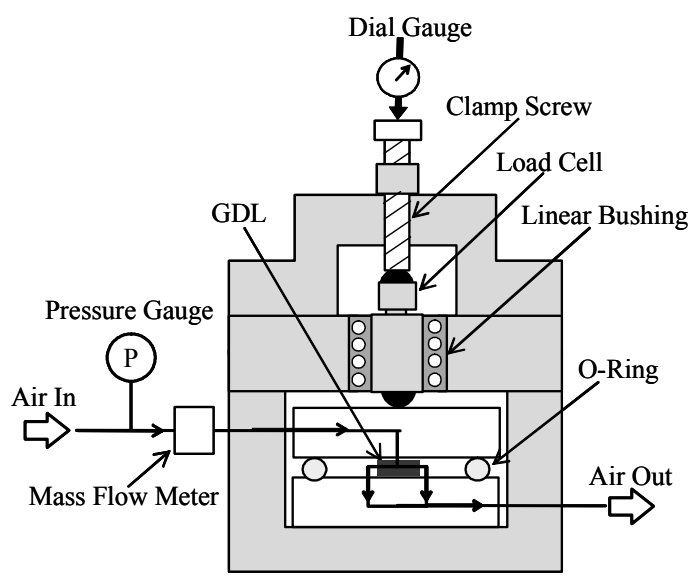

Fig.1 Schematic diagram of GDL permeability measurement apparatus

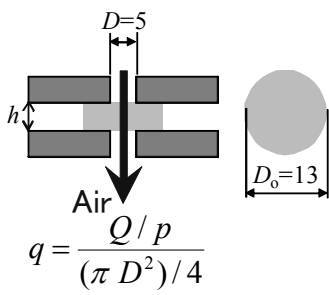

(a) Through-plane

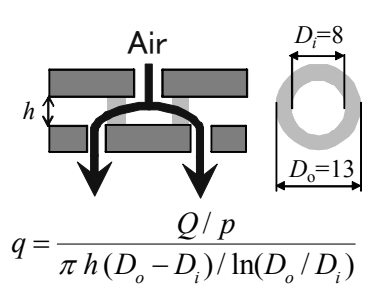

(b) In-plane

Fig.2 Major dimensions of GDL used for measuring air permeability in through-plane and in-plane directions 
The dry flow curve represents the relationship between the flow rate and the supplied air pressure obtained with a completely dry GDL and the wet flow curve represents that obtained with a wetted GDL in which a low-surface-tension $\quad(=0.0157 \mathrm{~N} / \mathrm{m})$ wetting liquid ${ }^{(12)}$ filled the pores of the GDL. The half-dry flow curve corresponds to one half the measured dry flow curve at a given air pressure.

By applying an air pressure across the wetted GDL, the liquid is displaced from the pores. The equilibrium relationship between the surface tension of the liquid and the air pressure, as shown in Figure 4, was used to calculate the pore diameter.

$$
d=\frac{4 \gamma \cos \theta}{P}
$$

where $d$ is pore diameter, $\gamma$ and $\theta$ are the surface tension and contact angle of the wetting liquid, respectively, $P$ is the air pressure acting on the liquid in the pore. The contact angle of the wetting liquid is close to $0^{\circ}{ }^{(12)}$. The minimum pressure at which air begins to flow by clearing of the first pore is the bubble point pressure. The maximum pore diameter $d_{\max }$ is calculated using this pressure. The pressure is further increased and progressively smaller pores are cleared until the GDL is fully dried. The mean flow pore diameter $d_{\mathrm{m}}$ is calculated using the mean flow pressure corresponding to the intersection of the wet flow and half-dry flow curves.

The cross-sectional view of the MPL coated GDL demonstrates that the MPL is not simply coated on the substrate surface, but penetrates deeply into the porous substrate. Because the boundary between the MPL and the substrate observed from the micrograph is neither clear nor uniform, it is difficult to measure the MPL thickness considering the penetration into the substrate. Therefore, the average thickness of the MPL $h_{\mathrm{MPL}}$ considering the penetration into the substrate was evaluated by a comparison of the measured in-plane permeance using GDLs with and without the MPL as shown in Figure $5^{(9)}$. The in-plane permeance $q$ of the MPL coated GDL is given as follows.

$$
q=\frac{q_{M P L} h_{M P L}+q_{s u b} h_{s u b}}{h}
$$

where $h$ is the total thickness of the GDL, $q_{\text {sub }}$ and $q_{\mathrm{MPL}}$ are the permeance of the substrate 
Table 1 PEFC test conditions

\begin{tabular}{|c|c|c|c|c|c|}
\hline $\begin{array}{c}\text { Cell } \\
\text { Temp. }\end{array}$ & $\begin{array}{c}\text { Hydrogen } \\
\text { Utilization }\end{array}$ & $\begin{array}{c}\text { Air } \\
\text { Utilization }\end{array}$ & $\begin{array}{c}\text { Relative Humidity } \\
\text { of Anode Inlet Gas }\end{array}$ & $\begin{array}{c}\text { Relative Humidity } \\
\text { of Cathode Inlet Gas }\end{array}$ & $\begin{array}{c}\text { Back } \\
\text { Pressure }\end{array}$ \\
\hline $75^{\circ} \mathrm{C}$ & $70 \%$ & $60 \%$ & $100 \%$ & $0 \%$ or $100 \%$ & $0 \mathrm{MPa}$ \\
\hline
\end{tabular}

and the MPL, respectively, and $h_{\text {sub }}$ and $h_{\text {MPL }}$ are the thickness of the substrate and the MPL, respectively. From Equation (2), the MPL thickness can be obtained as follows.

$$
h_{M P L}=\frac{h}{(1-k)}\left(1-\frac{q}{q_{\text {sub }}}\right)
$$

where $k\left(=q_{\mathrm{MPL}} / q_{\mathrm{sub}}\right)$ is the in-plane permeance ratio of the MPL to the substrate. The in-plane permeance ratio could not be obtained directly. In the present study, the values of $k$ were set at either $1 / 80$ for a mean flow pore diameter of $3 \mu \mathrm{m}$ or $1 / 150$ for a mean flow pore diameter of $1 \mu \mathrm{m}$, assuming that the in-plane permeance ratio was equal to the through-plane permeance ratio, which was measured using the substrate and the MPL coated GDL with a MPL thickness of $110 \mu \mathrm{m}$. Evidence for the through-plane permeance is presented later in the paper (see Figure 8).

\subsection{PEFC Performance Test}

The conditions of PEFC performance tests are summarized in Table 1. The cell temperature was set to $75{ }^{\circ} \mathrm{C}$. The utilization of hydrogen at the anode was set at $70 \%$ and the utilization of air at the cathode was set at $60 \%$. The relative humidity of the supplied gas at the cathode was set at either $100 \%$ or $0 \%$, while maintaining a relative humidity of $100 \%$ at the anode. The back pressure of the supplied gases was set at zero. The active area of the MEA (PRIMEA ${ }^{\circledR} 5580$ ) was $4.2 \mathrm{~cm}^{2}$. The IR (ohmic loss), activation and concentration overpotentials were measured separately as follows ${ }^{(13)}$.

(a) Anode overpotential $\left(\eta_{\mathrm{A}}\right)$ was obtained as the difference between the anode cell voltage and the reference hydrogen electrode.

(b) Open circuit voltage $(\mathrm{OCV})$ overpotential $\left(\eta_{\mathrm{OCV}}\right)$ was measured as the potential drop between the Nernst potential $\left(E_{0}\right)$ and OCV.

(c) IR overpotential $\left(\eta_{\mathrm{IR}}\right)$, or ohmic loss, was measured using the current-interruption method.

(d) Cathode concentration overpotential $\left(\eta_{\mathrm{CC}}\right)$ was determined by separating the voltage given by Tafel's equation from the cathode overpotential.

(e) Cathode activation overpotential $\left(\eta_{\mathrm{CA}}\right)$ was calculated by subtracting the above overpotentials and the output voltage ( $\left.V_{\text {eff }}\right)$ from $E_{0}$ as follows:

$$
\eta_{C A}=E_{0}-\eta_{A}-\eta_{e f f}-\eta_{C C}-\eta_{O C V}-\eta_{I R}
$$

The differences in the activation overpotentials obtained for all GDLs were not significant. Therefore, the influences of the MPL design parameters on the IR and concentration overpotentials were discussed in the following test results. Further study is required to clarify the influence of the MPL coated GDL on the activation overpotential.

\subsection{Gas Diffusion Layers}

The GDL substrate was a commercial carbon paper (SGL SIGRACET ${ }^{\circledR} 24 \mathrm{BA}$ ) that was treated by $5 \%$ PTFE (polytetrafluoroethylene) loading to impart hydrophobicity. The substrate has a thickness of $190 \mu \mathrm{m}$, an areal weight of $54 \mathrm{~g} / \mathrm{m}^{2}$, a porosity of $84 \%$ and a mean flow pore diameter of $42 \mu \mathrm{m}$. The MPL, which consists of $20 \%$ PTFE and $80 \%$ carbon black, was coated on the substrate as follows. A slurry containing PTFE, carbon 
black, distilled water and a surface-active agent was mixed using an impeller blade-type mixer and then spread on the substrate using a bar coating machine. The MPL was dried in an oven and then finally heated at $350^{\circ} \mathrm{C}$ to remove any remaining the solvent and to sinter the PTFE-binder and carbon black on the substrate. Figures 6 and 7 show surface and cross-sectional views of the GDLs with and without MPLs, respectively. The mean flow pore diameter $d_{\mathrm{m}}$ of the MPL coated GDL decreased from $10 \mu \mathrm{m}$ to $1 \mu \mathrm{m}$ when the water concentration in the slurry was reduced. The MPL thickness $h_{\mathrm{MPL}}$ considering the penetration in the substrate was controlled to between 90 and $240 \mu \mathrm{m}$ by the clearance and pressure of the doctor blade in the coating machine. The total thickness $h$ of all MPL coated GDLs was set at $250 \mu \mathrm{m}$.

\section{Results and Discussion}

\subsection{Influence of the MPL on Through-plane and In-plane Permeability}

Figure 8 shows the influence of the mean flow pore diameter $d_{\mathrm{m}}$ on the through-plane and in-plane permeance. The MPL thickness $h_{\mathrm{MPL}}$ was set at $110 \mu \mathrm{m}$ for all GDLs. The through-plane permeance obtained with the MPL coated GDL (black circle) is lower than that with the GDL substrate without the MPL (white circle). A decrease in the MPL pore diameter reduces through-plane permeability significantly, but reduces in-plane permeability only slightly. Figure 9 shows the influence of the MPL thickness $h_{\mathrm{MPL}}$ on the through-plane and in-plane permeance. The mean flow pore diameter $d_{\mathrm{m}}$ was set at $3 \mu \mathrm{m}$ for

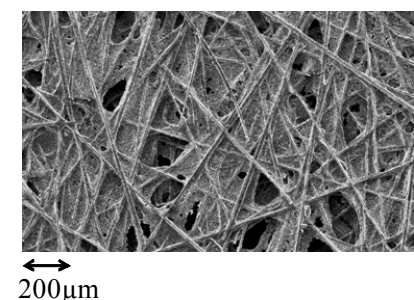

(a) Substrate $\left(24 \mathrm{BA}, d_{\mathrm{m}}=42 \mu \mathrm{m}\right)$

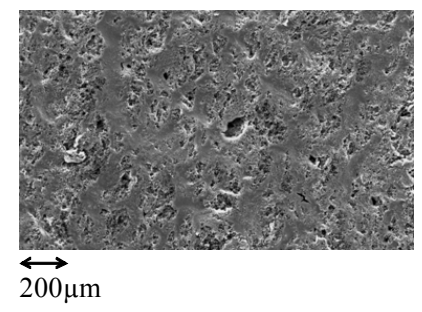

(b) GDL with MPL $\left(d_{\mathrm{m}}=10 \mu \mathrm{m}\right)$

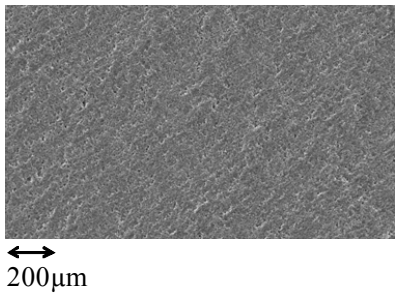

(c) GDL with MPL $\left(d_{\mathrm{m}}=1 \mu \mathrm{m}\right)$

Fig.6 Surface views of GDLs with and without MPL

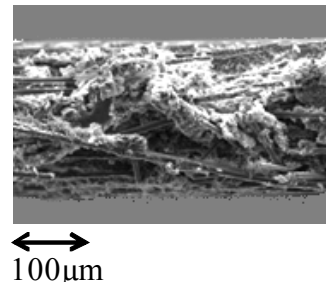

(a) Substrate $(24 \mathrm{BA}, h=190 \mu \mathrm{m})$

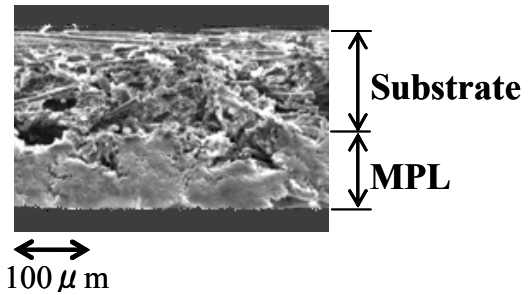

(b) GDL with MPL $\left(h_{\mathrm{MPL}}=110 \mu \mathrm{m}, h=250 \mu \mathrm{m}\right)$

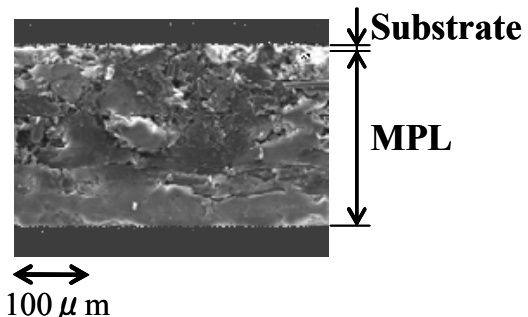

(c) GDL with MPL $\left(h_{\mathrm{MPL}}=240 \mu \mathrm{m}, h=250 \mu \mathrm{m}\right)$

Fig.7 Cross-sectional views of GDLs with and without MPL 


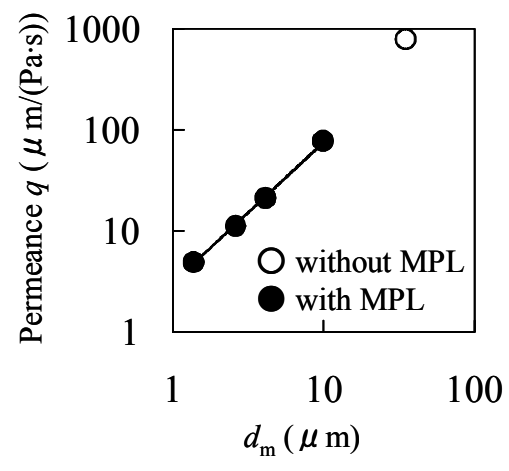

(a) Through-plane

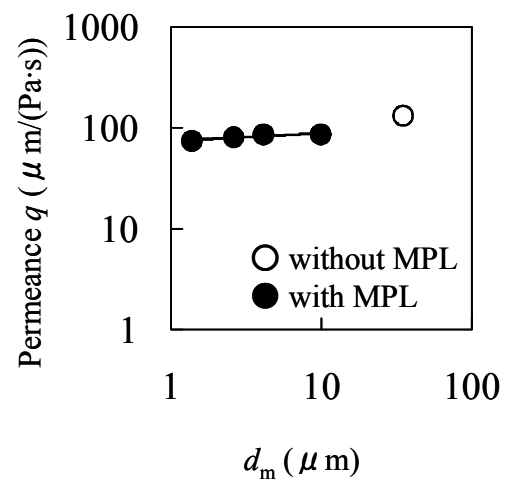

(b) In-plane

Fig. 8 Influence of mean flow pore diameter on GDL permeability $\left(h_{\mathrm{MPL}}=110 \mu \mathrm{m}\right)$

all GDLs. When the MPL thickness decreases, the in-plane permeance is enhanced, but enhancement of the through-plane permeance is not significant.

For the through-plane permeability tests, all of the supplied air flows out through the dense MPL, so that any decrease in the MPL pore diameter significantly reduces the through-plane permeability. For the in-plane permeability tests, although the MPL penetrates into the substrate, most of the supplied air flows out through the porous GDL substrate. Therefore, the influence of the MPL pore diameter on in-plane permeability is not significant. A decrease in the MPL thickness penetrated into the substrate increases the porous substrate cross-sectional area, which significantly enhances the in-plane permeability.

Figure 10 shows the relationship between the through-plane permeance $q$, porosity $\varepsilon$ and mean flow pore diameter $d_{\mathrm{m}}$ obtained using GDLs with and without MPLs. For the tested GDLs without the MPL, the porosity was varied between 83 and $91 \%$ and the thickness was varied between 190 and $300 \mu \mathrm{m}$. For the tested GDLs with the MPL, the MPL mean flow pore diameter was varied between 1 and $10 \mu \mathrm{m}$ and the MPL thickness was varied between 90 and $180 \mu \mathrm{m}$. Permeance increases in proportion to the porosity multiplied 
by the squared mean flow pore diameter $\left(\varepsilon d_{\mathrm{m}}{ }^{2}\right)$ for all GDLs without MPLs (white circles) ${ }^{(9)}$. Although the GDL has a very complex porous structure, a familiar relationship for a porous material consisting of straight capillary tubes with a porosity of $\varepsilon$ and a diameter of $d_{\mathrm{m}}$ can be obtained. On the other hand, for the MPL coated GDLs, there is no appropriate method to evaluate the porosity of the MPL. However, if the same relationship between permeance and $\varepsilon d_{\mathrm{m}}{ }^{2}$ is assumed, then the porosity of the MPL can be evaluated using the measured permenace and mean flow pore diameter ${ }^{(9)}$. Figure 11 demonstrates that the porosity of the MPL decreases from 50\% to $35 \%$ when the MPL mean flow pore diameter is reduced from $10 \mu \mathrm{m}$ to $1 \mu \mathrm{m}$.

\subsection{Influence of the MPL on PEFC Performance under High Humidity}

Figure 12 shows the influence of the MPL on the PEFC performance under high humidity when the mean flow pore diameter $d_{\mathrm{m}}$ of the cathode GDL is varied between 1 and $10 \mu \mathrm{m}$. The relative humidity of the supplied gases at both the anode and cathode was set to $100 \%$. The 24BA GDL without MPL was used at the anode. The performance obtained using the 24BA GDL without the MPL at the cathode is low due to flooding caused by the product water, which inhibits the transport of oxygen to the electrode and increases the concentration overpotential $\eta_{\mathrm{CC}}$ at high current densities. The performance obtained using the MPL coated GDLs were superior to the 24BA GDL without the MPL, due to the lower concentration overpotential $\eta_{\mathrm{CC}}$, although the IR overpotential $\eta_{\mathrm{IR}}$ was relatively uniform for all GDLs. The MPL coating is effective for enhancement of the PEFC performance, which varies significantly depending on the MPL

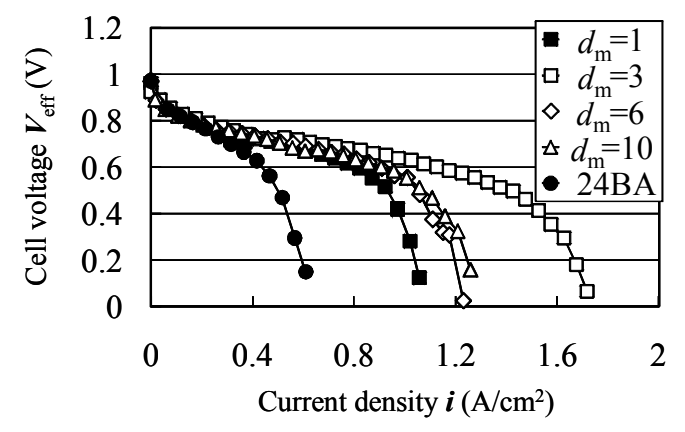

(a) Output voltage

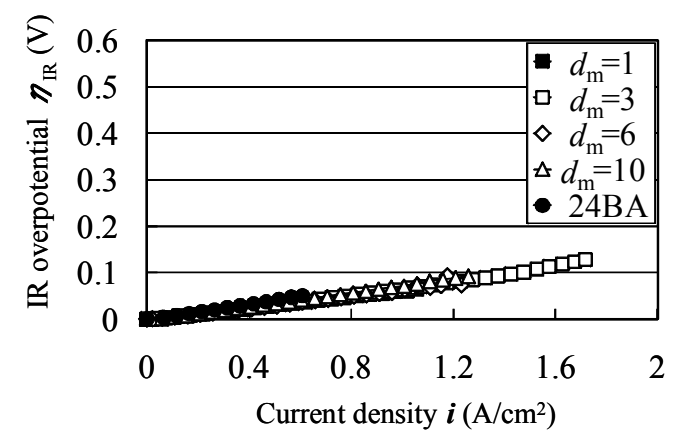

(b) IR overpotential

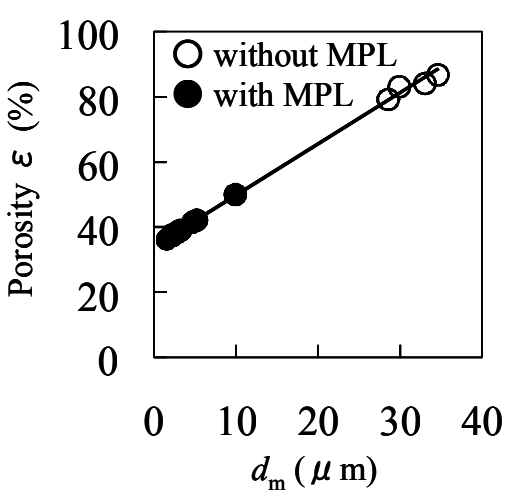

Fig.11 Relationship between porosity and mean flow pore diameter obtained using GDLs with and without MPL

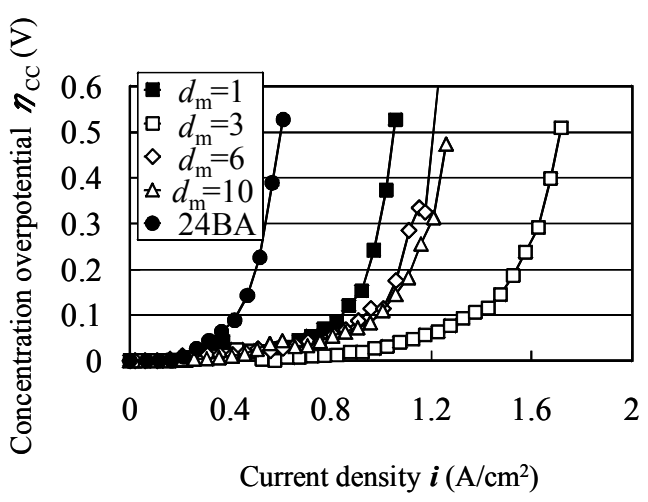

(c) Concentration overpotential

Fig.12 Influence of mean flow pore diameter of cathode GDL on PEFC performance under high humidity $\left(h_{\mathrm{MPL}}=110 \mu \mathrm{m}\right)$ 
pore diameter. Decreasing the pore diameter to $3 \mu \mathrm{m}$ is effective to reduce flooding and enhance PEFC performance. However, when the pore diameter becomes too small, the PEFC performance tends to decrease.

The best performance was obtained for the mean flow pore diameter of $3 \mu \mathrm{m}$. It is considered that an appropriate MPL coating between the electrode and the substrate reduces flooding on the cathode electrode, as shown in Figure 13. The water vapor produced from the electrochemical reaction at the cathode electrode is expelled through the MPL to the substrate. When the water vapor is condensed in the substrate, the water droplets accumulate in a large portion of the substrate pores. For the pore diameter of $3 \mu \mathrm{m}$, most of the liquid water is expelled to the gas channel in the separator, because the MPL is effective for preventing the back transport of liquid water from the substrate to the electrode. This is effective for a reduction in flooding at the cathode electrode ${ }^{(14)}$. However, when the MPL pore diameter is too large, the water droplets in the substrate are transported readily from the substrate to the electrode, resulting in an increase in the accumulation of liquid water at the cathode electrode. This promotes flooding at the cathode electrode, thereby lowering PEFC performance, as shown in Figure 12. When the MPL pore diameter is too small, gas permeability is significantly decreased, and the transport of the water vapor generated from the electrode to the substrate is inhibited and the diffusion of oxygen gas to the electrode is also reduced. This increases the concentration overpotential, thereby lowering PEFC performance.

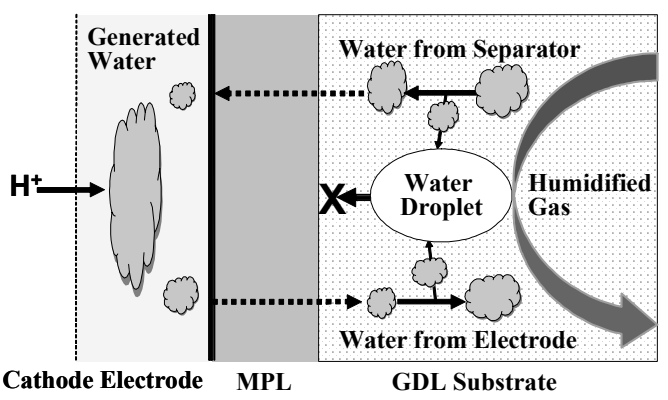

Fig.13 Appropriate MPL to prevent flooding at cathode electrode

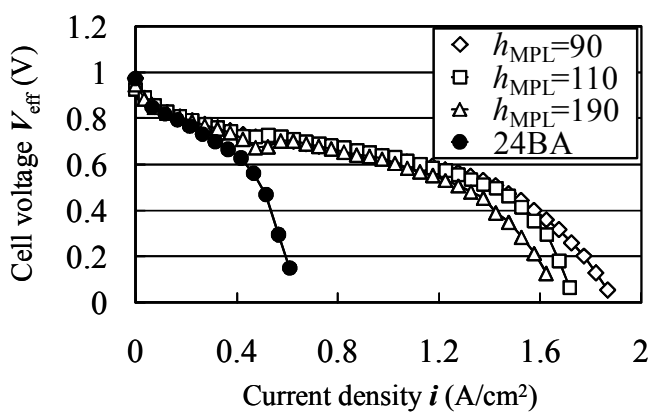

(a) Output voltage

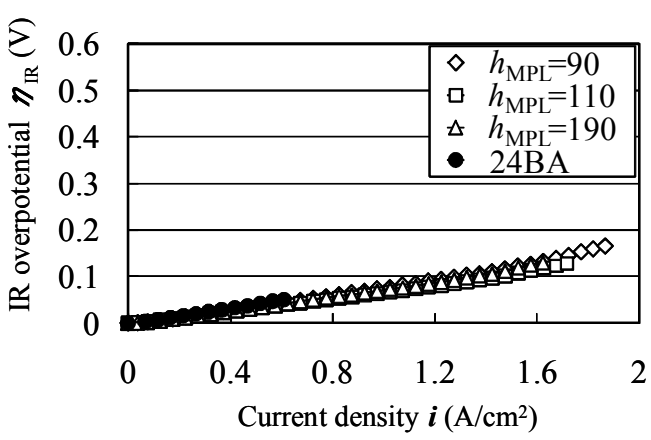

(b) IR overpotential

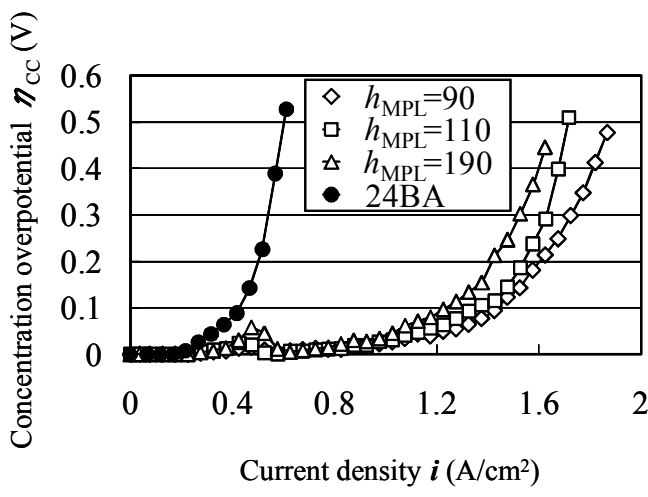

(c) Concentration overpotential

Fig.14 Influence of MPL thickness of cathode GDL on PEFC performance under high humidity $\left(d_{\mathrm{m}}=3 \mu \mathrm{m}\right)$ 
Figure 14 shows the influence of the MPL on the PEFC performance under high humidity when the MPL thickness $h_{\mathrm{MPL}}$ of the cathode GDL is varied between 90 and 190 $\mu \mathrm{m}$. The mean flow pore diameter of $3 \mu \mathrm{m}$, which displayed excellent performance (Figure 12), was applied to all MPLs. Reducing the MPL thickness improves in-plane permeability, which is effective for the further enhancement of the ability to prevent flooding.

\subsection{Influence of the MPL on PEFC Performance under Low Humidity}

Figure 15 shows the influence of the MPL on the PEFC performance under low humidity when the mean flow pore diameter $d_{\mathrm{m}}$ of the cathode GDL is varied between 1 and $10 \mu \mathrm{m}$. The relative humidity of the supplied gas at the cathode was set to $0 \%$, while maintaining a relative humidity of $100 \%$ at the anode. The 24BA GDL without MPL was used at the anode ${ }^{(8)}$. The IR and concentration overpotentials for the MPL coated GDLs were lower compared for the 24BA GDL without MPL, which indicates that the MPL is effective for enhancement of the PEFC performance under low humidity. Even when the relative humidity of the air supplied to the cathode is zero, water is transported into the MEA from the humidified hydrogen gas by electro-osmotic drag, and is also produced from the electrochemical reaction at the cathode. The wetness of the MEA is affected by the water balance between the conservation of membrane humidity and the discharge of water at the cathode. In the case of the cathode GDL without MPL, dehydration of the MEA caused by dry air at the cathode cannot be avoided. When the water content in the MEA becomes low, the ionic conductivity is reduced, which results in an increased IR overpotential. Moreover, the decrease in the water content of the MEA reduces the amount of protons transported from the anode to the cathode. This reduces the effective reaction area at triple phase boundaries, that is, the intersection of the ion-conductive electrolyte, the electrically conductive electrode and the gas-phase pores, which results in an increased concentration overpotential $^{(8)}$. The ability of the MPL to prevent dehydration of the MEA varies significantly, depending on the MPL pore

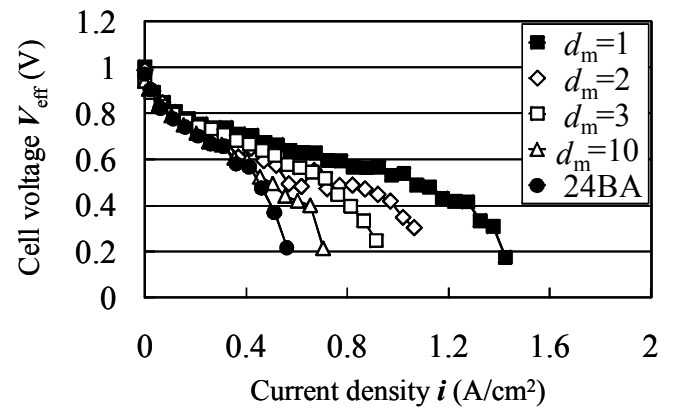

(a) Output voltage

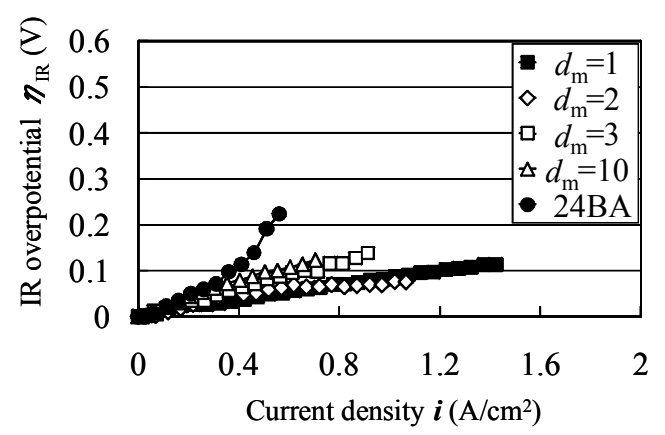

(b) IR overpotential

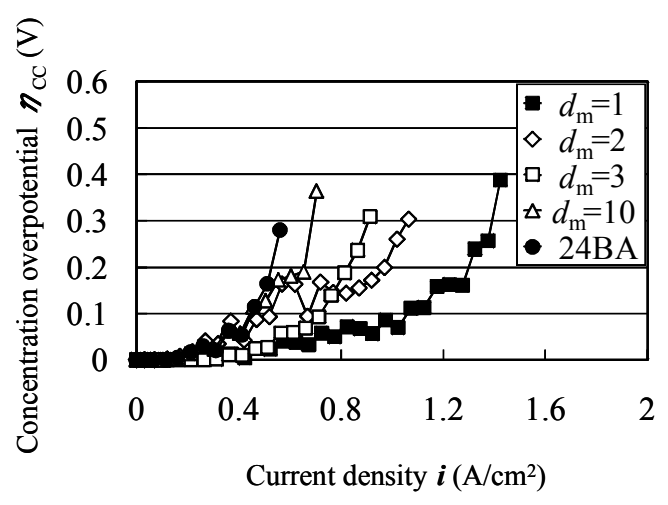

(c) Concentration overpotential

Fig. 15 Influence of mean flow pore diameter of cathode GDL on PEFC performance under no -humidification at the cathode $\left(h_{\mathrm{MPL}}=110 \mu \mathrm{m}\right)$ 
diameter. Decrease in the MPL pore diameter reduces the through-plane permeability, so that it is difficult for the MEA water to be expelled to the GDL substrate where it can be removed via dry air in the separator gas channel. This enhances the ability of the MPL to prevent dehydration of the MEA.

Figure 16 shows the influence of the MPL on the PEFC performance under low humidity when the MPL thickness $h_{\mathrm{MPL}}$ of the cathode GDL is varied between 110 and $240 \mu \mathrm{m}$. The mean flow pore diameter of $1 \mu \mathrm{m}$, which displayed excellent performance (Figure 15), was applied to all MPLs. Increasing the MPL thickness reduces both the through-plane and in-plane permeability of the GDL. This is effective for maintaining the humidity of the MEA. However, when the MPL thickness becomes too large, the transport of oxygen to the electrode through the MPL is decreased, which increases the concentration overpotential, thereby lowering PEFC performance.

\section{Conclusions}

The influence of MPL design parameters on gas permeability and PEFC performance under high and low humidity was investigated and the following conclusions were obtained.

(1) Decrease in the MPL pore diameter significantly reduces through-plane permeability, but reduces in-plane permeability only slightly. In-plane permeability of the MPL coated GDL is significantly enhanced by reducing the MPL thickness penetrated into the substrate.

(2) The permeance increases in proportion to the porosity multiplied by the squared mean flow pore diameter $\left(\varepsilon d_{\mathrm{m}}{ }^{2}\right)$ for the GDLs without the MPL. If the same relationship between the permeance and $\varepsilon d_{\mathrm{m}}{ }^{2}$ for the MPL coated GDLs is assumed, the porosity of the MPL can be evaluated using the measured permeance and mean flow pore diameter.

(3) Under high humidity, a decrease in the MPL pore diameter is effective to reduce flooding, which enhances PEFC performance. However, when the pore diameter becomes too small, PEFC performance tends to decrease. The best performance is obtained at the mean flow pore diameter of $3 \mu \mathrm{m}$. Reduction in the MPL thickness improves in-plane permeability and enhances the ability of the MPL to prevent flooding. 
(4) Under low humidity, decreasing the mean flow pore diameter to $1 \mu \mathrm{m}$ is effective to prevent dehydration of the MEA. Increasing the MPL thickness is also effective for maintaining the humidity of the MEA. However, when the MPL thickness becomes too large, the transport of oxygen to the electrode through the GDL is decreased, which lowers PEFC performance.

\section{References}

(1) Murata, M., Fuel cell separator and gas diffusion media (in Japanese), Journal of Society of Automotive Engineers of Japan, Vol. 59, No.2 (2005), pp. 109-112.

(2) Qi, Z. and Kaufman, A., Improvement of water management by a microporous sublayer for PEM fuel cells, Journal of Power Sources, Vol. 109 (2002), pp. 38-46.

(3) Wilde, P. M., Mandle, M., Murata, M. and Berg, N., Structural and physical properties of GDL and GDL / BPP combinations and their influence on PEMFC performance, Fuel Cells, Vol.4, No.3 (2004), pp.180-184.

(4) Chen, J., Matsuura, T. and Hori, M., Novel gas diffusion layer with water management function for PEMFC, Journal of Power Sources, Vol. 131 (2004), pp.155-161.

(5) Park, G. G., Sohn, Y. J., Yang, T.H., Yoon, Y.G., Lee, W.Y. and Kim, C.S., Effect of PTFE contents in the gas diffusion media on the performance of PEMFC, Journal of Power Sources, Vol. 131 (2004), pp.182-187.

(6) Konomi, T., Kitahara, T. and Sasaki, Y., Effect of design parameters in paper type GDL on PEFC performance (Effects of hydrophobic treatment, micro porous layer and specific density on performance) (in Japanese), Transactions of the Japan Society of Mechanical Engineers, Series B, Vol.72, No.715 (2006), pp.758-763.

(7) Murata, M., Kitahara, T., Konomi, T. and Nakajima, H., Effects of design parameters in paper type gas diffusion layer on the performance of polymer electrolyte fuel cells (Measures to prevent flooding and drying-up) (in Japanese), Transactions of the Japan Society of Mechanical Engineers, Series B, Vol.74, No.737 (2008), pp.183-189.

(8) Kitahara, T., Konomi, T., Nakajima, H., Tateishi, Y., Murata, M., Haak, N. and Wilde, P., Best combination of gas diffusion layers for polymer electrolyte fuel cell under cathode condition of very low humidity, ECS Transactions, Vol. 16, No.2 (2008), pp.1603-1613.

(9) Kitahara, T., Konomi, T., Nakajima, H. and Tateishi, Y., Influences of gas diffusion layer permeability on the performance of polymer electrolyte fuel cells (in Japanese), Transactions of the Japan Society of Mechanical Engineers, Series B, Vol.74, No.746 (2008), pp.2221-2228.

(10) Kitahara, T. and Konomi, T., Effect of clamp load on PEFC performance and over voltage (Comparison of contact pressure between paper and cloth type GDLs) (in Japanese), Transactions of the Japan Society of Mechanical Engineers, Series B, Vol.72, No.716 (2006), pp.1007-1012.

(11) Japanese Industrial Standards Committee, Paper and board - Determination of air permeance - Gurley method (in Japanese), JIS P 8117 (1998), pp.1-13.

(12) Jena, A. and Gupta, K., An innovative technique for pore structure analysis of fuel cell and battery components using flow porometry, Journal of Power Sources, Vol. 96 (2001), pp. 214-219.

(13) Konomi, T. and Sasaki, Y., Effects of running condition on over voltage in PEFC (in Japanese), Transactions of the Japan Society of Mechanical Engineers, Series B, Vol.71, No.705 (2005), pp.1428-1435.

(14) Konomi, T., Kitahara, T. and Iwata, Y., Analysis of water balance in PEFC at transient operation mode (Effects of hydrophobic treatment and micro porous layer on PEFC performance at starting test procedure mode) (in Japanese), Transactions of the Japan Society of Mechanical Engineers, Series B, Vol.72, No.715 (2006), pp.764-770. 